\title{
Blastic plasmacytoid dendritic cell neoplasm: A case report and literature review
}

\author{
YI-WEI ZHANG, JI-HUA ZHONG, XIAO-LONG CHEN, FEI XIAO and FANG-YUAN CHEN
}

Department of Hematology, Renji Hospital, School of Medicine, Shanghai Jiao Tong University, Shanghai 200127, P.R. China

Received October 27, 2014; Accepted September 24, 2015

DOI: $10.3892 / e t m .2016 .3259$

\begin{abstract}
Blastic plasmacytoid dendritic cell neoplasm (BPDCN) is a clinically aggressive tumor, which frequently presents as cutaneous lesions and subsequently progresses to bone marrow (BM) involvement and leukemic dissemination. BPDCN is a rare entity that belongs in the same class as acute myeloid leukemia-associated precursor neoplasms, according to the 2008 World Health Organization classification. The present study reported the case of a 26-year-old female who presented with evident thrombocytopenia, leukocytosis and anemia, but without skin lesions. The results of peripheral blood, BM smear and BM biopsy examinations detected numerous blastic or abnormal cells. In addition, flow cytometric analysis of BM demonstrated the presence of plasmacytoid dendritic cell-neoplastic precursor cells $\left(\mathrm{CD} 4^{+}\right.$, $\mathrm{CD}^{+}{ }^{+}, \mathrm{CD} 23^{+}, \mathrm{CD} 304^{+}$and human leukocyte antigen-DR ${ }^{+}$ phenotype).
\end{abstract}

\section{Introduction}

Blastic plasmacytoid dendritic cell neoplasm (BPDCN) is a rare entity that was initially identified in 1994 (1). However, the lack of knowledge regarding the histogenesis of BPDCN led to a succession of different classifications of this disease as a tumor, blastic natural killer (NK) leukemia/lymphoma, agranular $\mathrm{CD}^{+} \mathrm{NK}$ cell leukemia or agranular $\mathrm{CD}^{+} \mathrm{CD}^{+} 6^{+}$ hematodermic neoplasm $(2,3)$. According to the 2008 World Health Organization (WHO) classification,BPDCN is included in the same class as the acute myeloid leukemia-associated precursor neoplasms (4). The disease is characterized by predominant cutaneous involvement with ensuing or concomitant spreading to the peripheral blood (PB) and the bone marrow (BM). BPDCN has an aggressive clinical behavior with poor survival rates.

Correspondence to: Dr Ji-Hua Zhong, Department of Hematology, Renji Hospital, School of Medicine, Shanghai Jiao Tong University, 1630 Dongfang Road, Shanghai 200127, P.R. China

E-mail: jhzhong28@163.com

Key words: plasmacytoid, dendritic cell, neoplasm
BPDCN is characterized by numerous cutaneous lesions at diagnosis, in addition to extracutaneous involvement of the bone marrow, peripheral blood and lymph nodes. Patients usually exhibit asymptomatic, single or numerous skin lesions, which may be nodules, plaques or bruise-like. Extracutaneous disease is observed in the majority of patients at diagnosis, frequently involving the regional lymph nodes. As the BPDCN progresses, the peripheral blood and bone marrow may be involved (5).

The present study reported the case of a 26-year-old BPDCN patient, who presented with thrombocytopenia, leukocytosis and anemia, but with no skin lesions.

\section{Case report}

In March 2014, a 26-year-old Chinese female was admitted to Renji Hospital (Shanghai, China) with pancytopenia. The patient presented with evident thrombocytopenia, leukocytosis and anemia. Laboratory results revealed a hemoglobin level of $5.6 \times 10^{10} \mathrm{~g} / 1$ (normal range, $11.0-15.0 \times 10^{10} \mathrm{~g} / \mathrm{l}$ ), white blood cell count of $2.83 \times 10^{9} / 1$ (normal range, $4-10 \times 10^{9} / 1$ ) and platelet count of $8.6 \times 10^{10} / 1$ (normal range, $10.0-30.0 \times 10^{10} / 1$ ). The patient did not have enlarged lymph nodes (LNs), but sternal tenderness was observed. Symptoms included headache, earache and cough, with no gum swelling or bleeding. No history of asymptomatic, solitary or multiple skin lesions, such as nodules, plaques or bruise-like lesions, was noted. Written informed consent was obtained from the patient.

The patient presented with evident thrombocytopenia, leukocytosis and anemia. Therefore, it was suspected that the patient had pathology of the hematopoietic and lymphoid tissues. PB and BM smears were performed, and the results demonstrated that blastic or abnormal cells accounted for $66 \%$ of the nucleated cells. As seen in Fig. 1, the abnormal BM cells were found to have a relatively moderate nuclear to cytoplasmic ratio, oval nuclear contours, numerous nuclei with incisura and rough chromatin. In addition, the majority of cells exhibited elongated agranular and vacuoles in the cytoplasm (Fig. 1). Cytochemical staining revealed that the cells were positive for periodic acid-Schiff and $\alpha$-naphthyl acetate esterase, but negative for peroxidase (Fig. 1) and naphthol AS-D chloroacetate esterase.

BM biopsy demonstrated a markedly hypercellular marrow, in which the number of diffuse proliferative blastic cells was significantly higher compared with the number of 

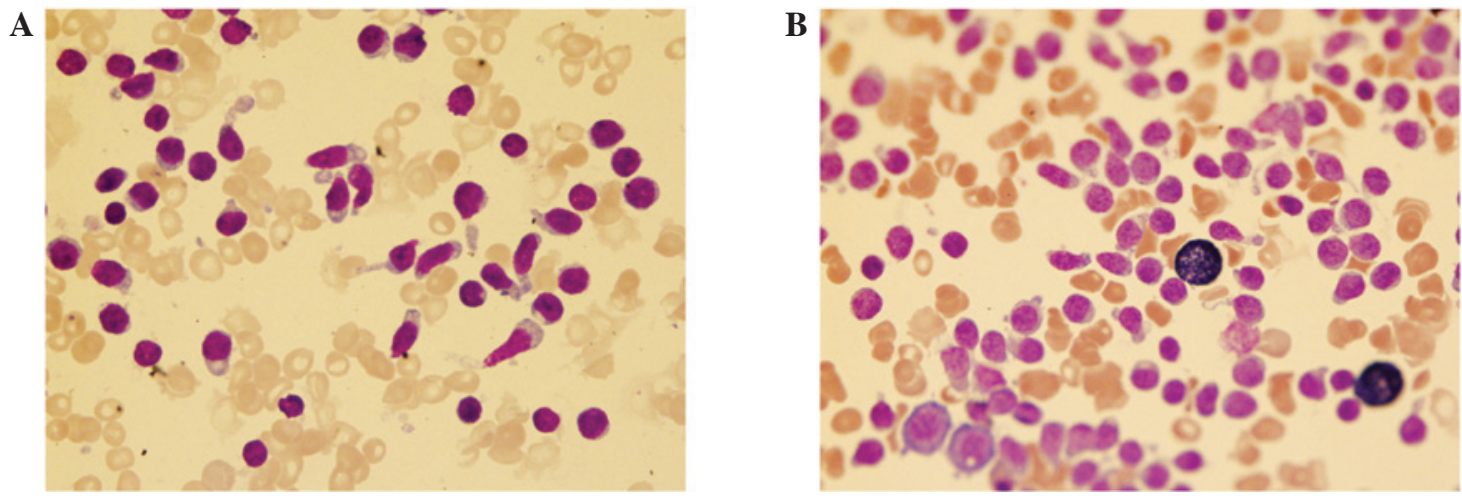

Figure 1. Bone marrow aspiration results. (A) Wright-Giemsa staining and (B) peroxidase staining (magnification, x1,000).
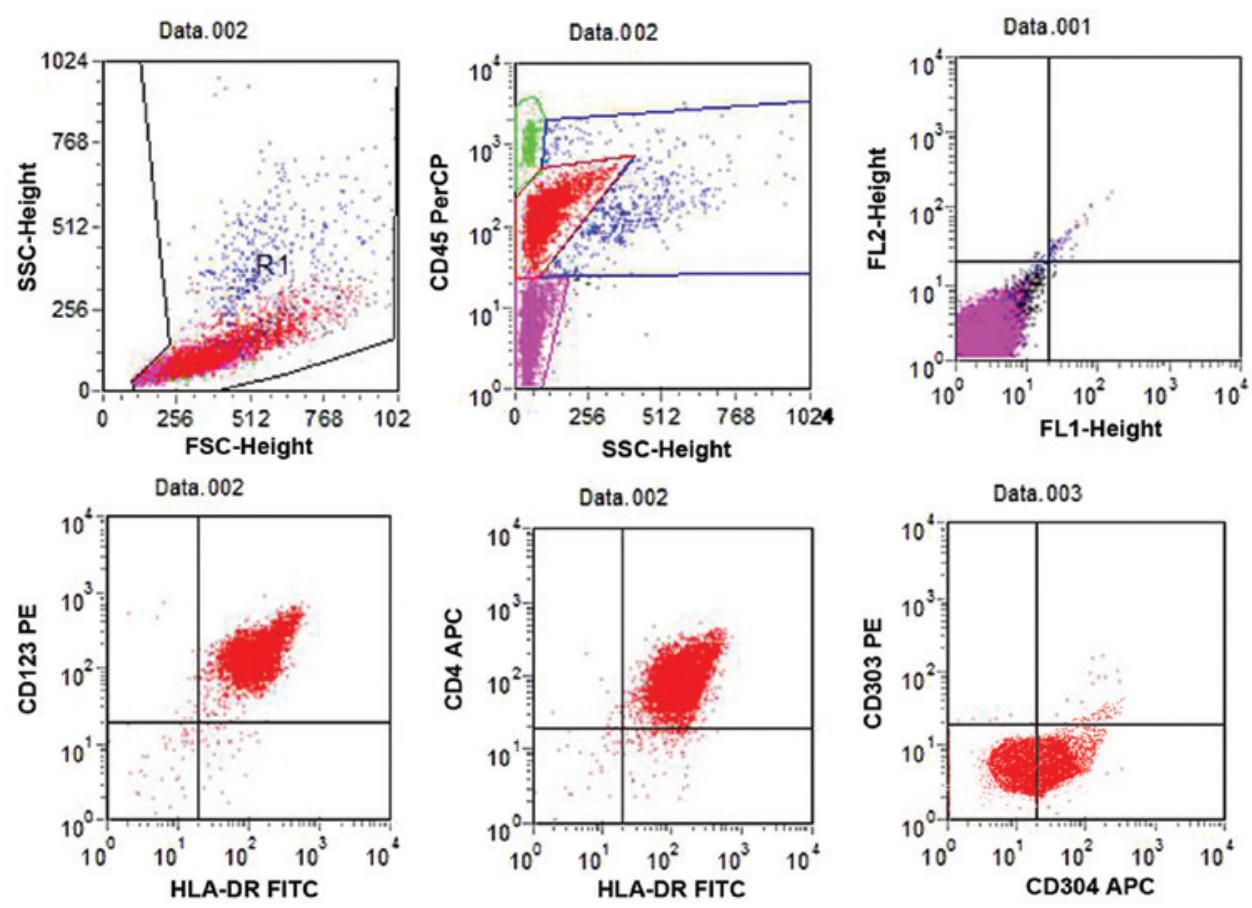

Figure 2. Flow cytometric immunophenotyping. Side scatter (SS) by CD45 flow cytometric profile of the blastic plasmacytoid dendritic cell neoplasm blast gate (red), demonstrating expression of CD4, CD123, HLA-DR and CD304. HLA-DR, human leukocyte antigen-DR.

normal cells. Based on the results of immunohistochemical analysis, the cells were found to co-express CD4 and CD56, while they were partially positive for terminal deoxynucleotidyl transferase and negative for myeloperoxidase (MPO), CD34, CD15, pericentriolar material 1, CD234, CD20, CD43 and $\mathrm{CD} 3$. In addition, the $\mathrm{Ki}-67$ proliferative index was $\sim 10 \%$ (reference value, $<20 \%$ ), which was within the normal range.

Flow cytometry was performed on the BM biopsy sample, and revealed $88.1 \%$ abnormal CD $45^{\text {dim }+}$ cells within the nuclear cell gate. In addition, the blastic plasmacytoid dendritic cells were positive for CD4 (61.6\%), CD7 (17.5\%), human leukocyte antigen (HLA)-DR (94\%), CD56 (99\%), CD123 (98\%) and CD304 (60\%), but negative for lineage-associated markers, including CD2, CD3, CD19, CD79a, CD11b, CD13, CD15, CD33, CD64, CD14, CD117, MPO, CD34, CD38, CD16, CD57, CD94, CD138 and CD303. Diagnosis of BPDCN was indicated by positivity for CD4 and CD56, along with other markers that are more restricted to plasmacytoid dendritic cells (such as CD123), and negativity for lymphoid, NK and myeloid lineage-associated antigens. Flow cytometry equipment (FACSCalibur ${ }^{\mathrm{TM}}$ ) and monoclonal antibodies (Pharmingen ${ }^{\mathrm{TM}}$ mouse anti-human) were purchased from BD Biosciences (San Diego, CA, USA).

The patient received a single course of daunorubicin, vincristine and prednisone. The treatment outcome was promising, with the patient appearing to go into remission and showing no symptoms at a follow-up examination in September 2015.

\section{Discussion}

BPDCN is a rare and aggressive hematodermic neoplasm, which typically occurs in elderly patients, with a mean age between 60 and 70 years; however, the disease may present at any age $(2,3,5)$. BPDCN is more prevalent in males, with a male to female ratio of $3: 1$. In addition, it is characterized by 
a high frequency of cutaneous lesions at diagnosis, which is accompanied by extracutaneous involvement of the LNs, PB and BM (6). Asymptomatic, solitary or multiple skin lesions, such as plaques, nodules or bruise-like lesions are commonly detected in these patients (7). BPDCN without cutaneous lesions typically occurs in young patients that also present evident leukocytosis anemia and thrombocytopenia, and the median survival time of these patients is shorter (6). PB and $\mathrm{BM}$ involvement is developed along with the disease progression. Low-level of BM and PB involvement is identified in the majority of cases (60-90\%); however, initial fulminant leukemia is rare $(5-25 \%)$. Furthermore, LN involvement at presentation is common $(40-50 \%)$, whereas splenomegaly $(<20 \%)$ or involvement of other mucosal sites $(<10 \%)$ are relatively rare. Systemic B symptoms are also rarely observed at diagnosis (3).

BPDCN can show diffuse involvement of the LNs and complete effacement of the LN architecture (3). It is characterized by the following features: A monotonous population of small to medium cells with irregular nuclear contours, fine to evenly dispersed chromatin, 1-3 small nucleoli, and scant to moderate amount of cytoplasm. The neoplastic cells can be present in the PB and resemble circulating leukemic lymphoid or myeloid blasts, particularly in cases with extensive involvement of the BM. The neoplastic cells in the BM aspirate tests may exhibit typical pearl necklace-like, submembranous, cytoplasmic vacuoles and elongated, agranular cytoplasm. Notably, the residual hematopoietic elements, including megakaryocytes, granulocytes and erythroid precursors, may present certain dysplastic alterations in the BM and in the PB (3). In the present case, the morphological features were in accordance with the typical features observed in BPDCN.

The diagnosis of BPDCN based on the results of immunohistochemical analysis requires positivity for CD4 and CD56, along with other markers that are more restricted to plasmacytoid dendritic cells (such as CD123), and negativity for lymphoid, NK and myeloid lineage-associated antigens $(8,9)$. BPDCN is typically identified in the CD45 $5^{\mathrm{dim}+}$ flow cytometry blast gate region; 'dim' indicates weakly positive detection. BPDCN is further phenotypically defined based on the absence of CD34 expression, the co-expression of CD56 and CD4, and the lack of specific myeloid-, T-, B- or NK-lineage markers (6,10-12). Furthermore, T-cell leukemia/lymphoma 1 (TCL1) and HLA-DR expression is typically observed, in addition to marked CD123 expression that is detected in the majority of cases, indicating the plasmacytoid dendritic cell origin of BPDCN. A previous study proposed novel markers and extended diagnostic criteria for BPDCN, including the detection of BDCA-2 (CD303) and BDCA-4 (CD304) expression (11).

Upon cutaneous presentation, differential diagnoses may include cutaneous T-cell or nasal-type NK lymphomas, myeloproliferative disorders with monocytic differentiation, or leukemia cutis (acute myeloid/lymphocytic leukemia) $(6,12)$. The diagnosis of BPDCN is facilitated when Epstein-Barr virus-positivity, absence of lineage-specific markers, specific dermatopathological features (including angio-invasion/-destruction in NK/T-cell lymphoma) and $\mathrm{T}$-cell receptor (TCR) gene rearrangements are observed (4).

In the present case, the diagnosis of BPDCN was challenging due to the absence of traditional lineage-specific markers and typical cutaneous presentation. However, the results of flow cytometric analysis identified the typical CD45 ${ }^{\mathrm{dim}+} \mathrm{CD} 4^{+}$ $\mathrm{CD} 6^{+} \mathrm{HLA}-\mathrm{DR}^{+} \mathrm{CD}_{123}{ }^{+} \mathrm{CD} 304^{+}$immunophenotype. These observations, along with the characteristic morphology and cytogenetic findings in the present case, indicated that the disease was consistent with the features of leukemic BPDCN. The main differential diagnosis for leukemic BPDCN is acute leukemia of ambiguous lineage, particularly the provisional entity NK-cell lymphoblastic leukemia/lymphoma (NK-LL/L) (4,13). Based on the 2008 WHO classification (4), in cases where BPDCN is excluded, the diagnosis of NK-LL/L may be considered when absence of B-cell or myeloid cell markers, negative TCR gene rearrangement and expression of CD56 with immature T-associated markers (such as cytoplasmic-CD3, CD7 and CD2) are observed (4). Clearly, BPDCN and NK-LL/L diagnoses may benefit from extended phenotypic panels that have been previously proposed (11). The characteristics observed in the present case are in accordance with the typical immunophenotypic features.

Patients with BPDCN usually respond well to initial chemotherapy, with stem cell transplantation in the pediatric population is reserved for patients who relapse. Outcomes are more favorable in cases that lack cutaneous disease at presentation, although a comparison of cutaneous and noncutaneous cases might be confounded by differences in treatment regimens. In future, it is imperative to identify the most effective treatment for all presentations of BPDCN, since the majority of cases are rapidly and uniformly fatal (14). The majority of the long-term survivors in the leukemic and cutaneous groups presented herein represented those who successfully underwent hematopoietic stem cell transplantation. Indeed, a recent study has suggested that allogeneic stem cell transplantation with reduced intensity conditioning should be pursued aggressively in BPDCN patients $(15,16)$.

\section{References}

1. Lencastre A, Cabete J, João A, Farinha P, Ferreira G and Lestre S: Blastic plasmacytoid dendritic cell neoplasm. An Bras Dermatol 88 (Suppl 1): 158-161, 2013.

2. Herling $\mathrm{M}$ and Jones D: $\mathrm{CD} 4^{+} / \mathrm{CD} 6^{+}$hematodermic tumor: The features of an evolving entity and its relationship to dendritic cells. Am J Clin Pathol 127: 687-700, 2007.

3. Feuillard J, Jacob MC, Valensi F, Maynadié M, Gressin R, Chaperot L, Arnoulet C, Brignole-Baudouin F, Drénou B, Duchayne E, et al: Clinical and biologic features of $\mathrm{CD} 4{ }^{+} \mathrm{CD} 56^{+}$ malignancies. Blood 99: 1556-1563, 2002.

4. Swerdlow SH, Campo E, Harris NL, Jaffe ES, Pileri SA Stein H, Thiele J and Vardiman JW: WHO Classification of Tumours of Haematopoietic and Lymphoid Tissues. 4th edition. International Agency for Research on Cancer (IARC), Lyon, 2008

5. Shi Y and Wang E: Blastic plasmacytoid dendritic cell neoplasm: a clinicopathologic review. Arch Pathol Lab Med 138: 564-569, 2014.

6. Petrella T, Bagot M, Willemze R, Beylot-Barry M, Vergier B, Delaunay M, Meijer CJ, Courville P, Joly P, Grange F, et al: Blastic NK-cell lymphomas (agranular CD4 ${ }^{+} \mathrm{CD} 56^{+}$hematodermic neoplasms): A review. Am J Clin Pathol 123: 662-675, 2005.

7. Reichard KK, Burks EJ, Foucar MK, Wilson CS, Viswanatha DS, Hozier JC and Larson RS: CD $4^{+} \mathrm{CD} 56^{+}$lineage-negative malignancies are rare tumors of plasmacytoid dendritic cells. Am J Surg Pathol 29: 1274-1283, 2005.

8. Facchetti F, Ungari M, Marocolo D, Lonardi S and Vermi W: Blastic plasmacytoid dendritic cell neoplasm. Haematol Meet Rep 3: 1-3, 2009. 
9. Pina-Oviedo S, Herrera-Medina H, Coronado H, Del Valle L and Ortiz-Hidalgo C: $\mathrm{CD} 4^{+} / \mathrm{CD} 6^{+}$hematodermic neoplasm: Presentation of 2 cases and review of the concept of an uncommon tumor originated in plasmacytoid dendritic cells expressing CD123 (IL-3 receptor alpha). Appl Immunohistochem Mol Morphol 15: 481-486, 2007.

10. Jegalian AG, Facchetti F and Jaffe ES: Plasmacytoid dendritic cells: Physiologic roles and pathologic states. Adv Anat Pathol 16: 392-404, 2009.

11. Garnache-Ottou F, Feuillard J, Ferrand C, Biichle S, Trimoreau F, Seilles E, Salaun V, Garand R, Lepelley P, Maynadié M, et al: Extended diagnostic criteria for plasmacytoid dendritic cell leukaemia. Br J Haematol 145: 624-636, 2009.

12. Herling $\mathrm{M}$ and Jones D: $\mathrm{CD}^{+} / \mathrm{CD} 6^{+}$hematodermic tumor: The features of an evolving entity and its relationship to dendritic cells. Am J Clin Pathol 127: 687-700, 2007.
13. Ham MF and Ko YH: Natural killer cell neoplasm: Biology and pathology. Int J Hematol 92: 681-689, 2010.

14. Jegalian AG, Buxbaum NP, Facchetti F, Raffeld M, Pittaluga S, Wayne AS and Jaffe ESL Blastic plasmacytoid dendritic cell neoplasm in children: Diagnostic features and clinical implications. Haematologica 95: 1873-1879, 2010.

15. Dietrich S, Andrulis M, Hegenbart U, Schmitt T, Bellos F, Martens UM, Meissner J, Krämer A, Ho AD and Dreger P: Blastic plasmacytoid dendritic cell neoplasia (BPDC) in elderly patients: Results of a treatment algorithm employing allogeneic stem cell transplantation with moderately reduced conditioning intensity. Biol Blood Marrow Transplant 17: 1250-1254, 2011.

16. Rauh MJ, Rahman F, Good D, Silverman J, Brennan MK, Dimov N, Liesveld J, Ryan DH, Burack WR and Bennett JM: Blastic plasmacytoid dendritic cell neoplasm with leukemic presentation, lacking cutaneous involvement: Case series and literature review. Leuk Res 36: 81-86, 2012. 